\title{
Epoch Description
}

National Cancer Institute

\section{Source}

National Cancer Institute. Epoch Description. NCI Thesaurus. Code C93824.

The textual representation of the epoch. 